\title{
FIB MICROSCOPY OF MARTIAN METEORITE ALH84001 CARBONATE DISKS
}

Michael P. Bernas ${ }^{1}$, Simon J. Clemett ${ }^{2}$, Kathie L. Thomas-Keprta ${ }^{2-3}$, David S. McKay ${ }^{4}$, Everett K. Gibson, Jr. ${ }^{4}$; ${ }^{1}$ FEI Company, 5350 NE Dawson Creek Drive, Hillsboro, OR 97124, ${ }^{2}$ ERC/NASA/JSC, Mail Code JE23, Houston, TX 77058, ${ }^{3}$ Texas Southern University, 3100 Cleburne Ave., Houston, TX 77004, ${ }^{4}$ NASA Johnson Space Center, Mail Code KA, Houston, TX 77058.

Introduction: Our aim is to understand the mechanism(s) of formation of carbonate assemblages in ALH84001. A prerequisite is that a detailed characterization of the chemical and physical properties of the carbonate be established. We present here analyses by transmission electron microscopy (TEM) of carbonate thin sections produced by both focused ion beam (FIB) sectioning and ultramicrotomy. Our results suggest that the formation of ALH84001 carbonate assemblages were produced by considerably more complex process(es) than simple aqueous precipitation followed by partial thermal decomposition as proposed by other investigators [e.g., 1-3].

Method: All the carbonate assemblages studied appeared as pseudo-spherical disks embedded within the orthopyroxene (Opx) matrix and were flush with the fracture surface (Fig 1). These were removed intact by careful application of pressure to the carbonate/Opx interface (and a certain amount of luck). A typical extracted assemblage was $\sim 200 \mu \mathrm{m}$ in diameter and $\sim 20 \mu \mathrm{m}$ thick. Electron transparent sections necessary for TEM analysis were prepared by two methods: (1) ultramicrotomy of epoxy embedded samples; and, (2) FIB sectioning of Ptcoated samples. Ultramicrotomy: $30-40 \mu \mathrm{m}$ size fragments from the intact carbonate disk were embedded in epoxy and sectioned with a $45^{\circ}$ diamond knife onto distilled water from which they were transferred to continuous $\mathrm{C}$ film TEM grid. Typical section thickness was 90-100 nm. FIB Sectioning: A complete carbonate disk was attached to a scanning electron microscope (SEM) mount by carbon adhesive tape and coated with $\sim 40 \mathrm{~nm}$ of Pt. Regions of interest identified by SEM were subsequently extracted using a FEI dual beam Strata $237 \mathrm{Ga}^{+}$FIB (for a more complete discussion of this technique see [4]); a typical FIB slab was $\sim 20 \times 7 \times 0.1 \mu \mathrm{m}$. Ultramicrotome and FIB sections were analyzed with a JEOL 2000 FX TEM equipped with a Thermo Noran System Six energy dispersive Xray spectrometer (EDS).

Results and Discussion: In ultramicrotomy the 'knife' that sections the sample is (in our case) actually a $45^{\circ}$ cleaved diamond wedge. This requires that as a section is cut it must be flexed to an equivalent angle at the point the knife intersects the sample. To obtain a contiguous, uniform thin section this requires the sample has a relatively low Young's modulus, be physically homogeneous, and structurally free of pre-existing fractures; unfortunately ALH84001 carbonate assemblages meet none of these criteria. Consequently carbonate microtome sections are typically fragmented and fail to preserve $\mu \mathrm{m}$-scale structural and textural relationships (Fig. 2). Furthermore, once a section has been cut by the diamond wedge, it is pushed into a distilled water bath and suspended by surface tension. Capillary action likely penetrates the sample resulting in possible contamination and/or relocalization of indigenous water soluble salts.

FIB sectioning uses a focused $\mathrm{Ga}^{+}$beam to sputter away selected regions of the sample to render an isolated slab that can subsequently be picked out of the sample using a micromanipulator and transferred directly to a continuous $\mathrm{C}$ film TEM mount. Since there is little or no structural flexing, the physical size of the section is not constrained by the same factors as microtomy (Fig. 2). Consequently, large slabs of electron transparent carbonate with a length-tothickness ratio $>300$ can be removed intact from the sample (Fig. 3). This allows the spatial location of the sample to both the carbonate and the Opx host matrix to be fully preserved (Fig.3). One potential disadvantage of the FIB technique is perturbation of the carbonate matrix surface by embedded Ga+ ions.

Sectioning by FIB reveals the carbonate to have a low porosity $(<20 \%)$, with parallel strain fractures and cross-cut with S-rich, grain filled veins. The chemical zonation of the carbonates occurs only along two axes and is invariant on the third, that is zoning propagates radially outward from the carbonate center but is constant through the thickness of the carbonate at a given radius (Fig. 4).

Void space appears throughout the carbonate, sometimes the presence of a void is accompanied by magnetite crystal(s), however we also observed fully encapsulated voids within the section containing no magnetite. Other investigators have suggested that void spaces with associated magnetite(s) were formed by thermal decomposition of Fe-carbonate to magnetite. We note that in many cases the void size associated with magnetite grains is significantly larger than could be explained solely by the density difference between magnetite and carbonate.

$\mathrm{A}$, as yet undetermined, Si-rich phase(s) is present homogeneously throughout the carbonate at $\sim 0.1-1.0 \mathrm{wt} . \%$ level. We cannot discount the presence of phyllosilicates and/or a Si-gel/glass.

Single crystal magnetite exist throughout the carbonate assemblages from core to rim but have the highest concentration in the optically dark outer rims. All magnetites characterized in the FIB sections were chemically pure, even when embedded within a carbonate matrix both Mg- and Mn-rich (Fig. 5). Both of these elements are capable of forming solid solutions of Mg- and Mn-ferrite, in particular incorporation of small amounts of Mn into magnetite may be thermodynamically favored due to the resulting lowering of the Curie temperature [5].

Implications: The use of FIB sectioning provides a hitherto unique view of the carbonate assemblages at the $\mathrm{nm}$ to $\mu \mathrm{m}$ scales in the absence of any embedding medium and under anhydrous conditions. The presence of carbonates inset into the Opx and flush with the fracture surface displaying cylindrically symmetrical zoning seems inconsistent with a simple aqueous precipitation process. Furthermore, the presence of the embedded magnetites within the sections studied here arguably requires one or more processes, other than thermal decomposition, to explain their origin.

This work supported in part by NASA Exobiology.

References: [1] Brearley A.J. (2003) MAPS 38, 849. [2] Treiman A.H. (2003) Astrobiology 3, 369. [3] Golden et al.(2004) Amer. Min. 89, 439. [4] Graham G.A. et al. LPSC XXXV, CD-ROM \#2044. [5] Isambert A. \& Valet J.-P. (2003) JGR 108 (B6) EPM 2-1. 


\section{Figure 1}

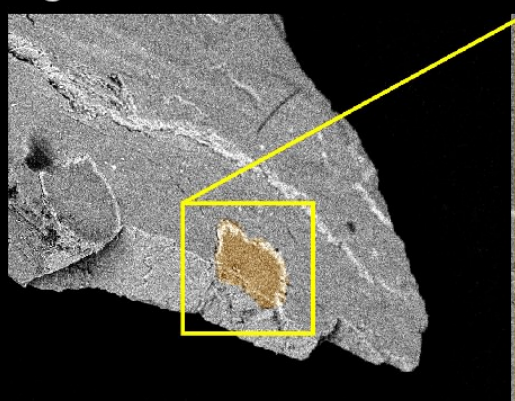

$50 \mu \mathrm{m}$

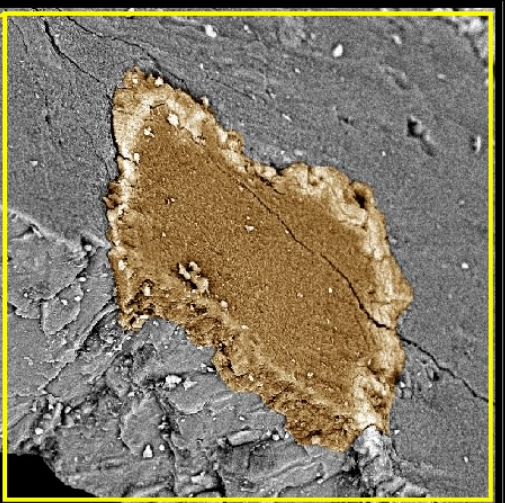

Figure 3
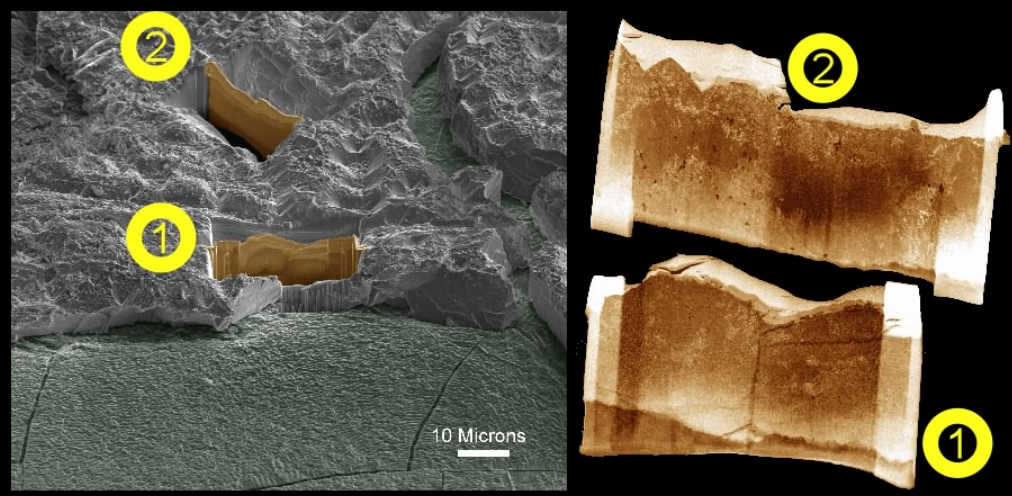

\section{Figure 5}
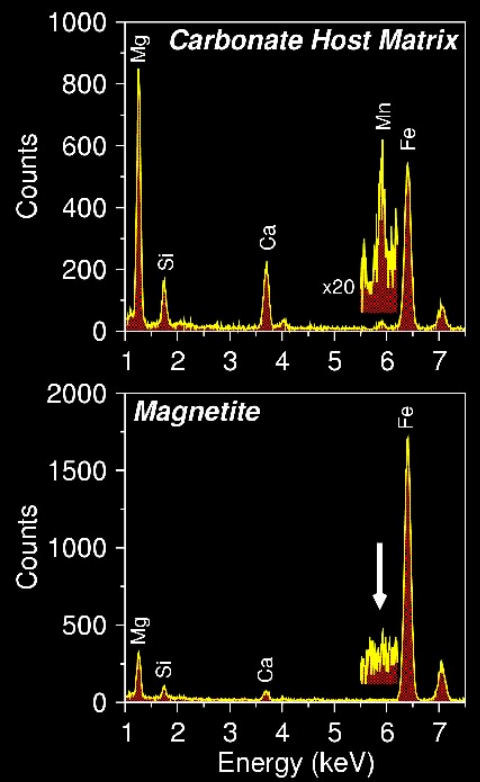
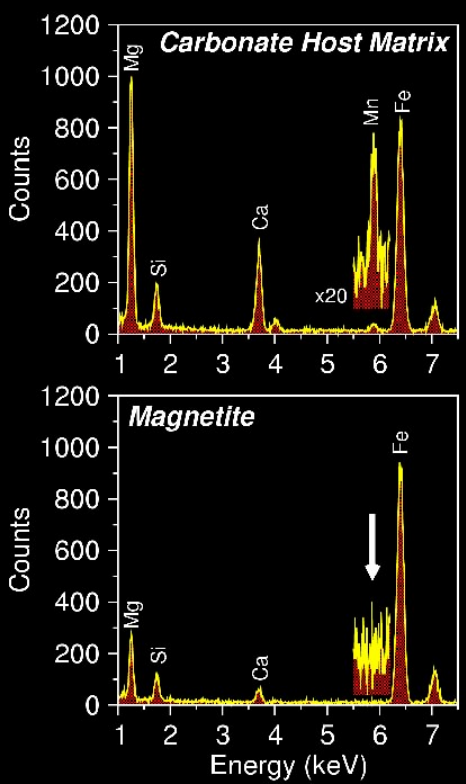

\section{Figure 2}

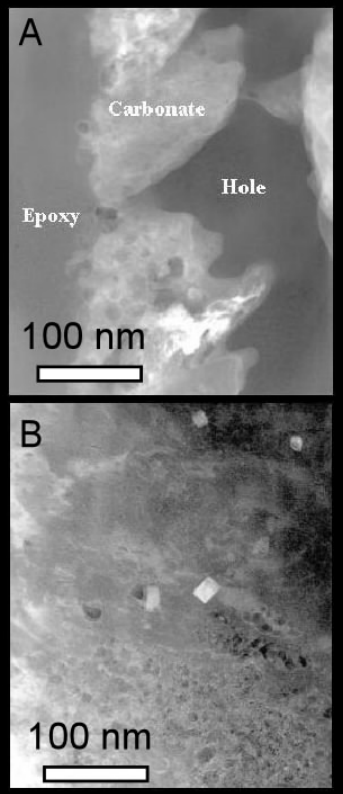

\section{Figure 4}

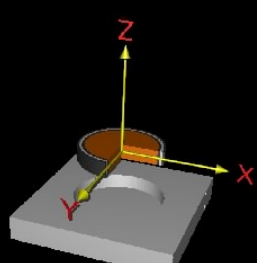

Figure 1. ALH84001 carbonate disk embedded within a form-fitting pit and flush with the Opx matrix surface.

Figure 2. Images of a typical ALH84001 carbonate microtome section (top) and FIB section (bottom). Note the lack of sample continuity in the microtome section.

Figure 3. False color SEM image (left) of two FIB pits (orange) in ALH84001 carbonate (gray) and corresponding TEM views of extracted FIB sections (right).

Figure 4. Cartoon of ALH84001 carbonate disk depicting $\mathrm{X}, \mathrm{Y}$ and $\mathrm{Z}$ directions. Cylindrical symmetrical chemical zoning occurs in the $\mathrm{X}-\mathrm{Y}$ plane. Carbonate composition is homogeneous through the thickness of the disk ( $\mathrm{Z}$ value) at any specific X-Y point. ALH84001 carbonate disks appear to be composed of vertical, rather than horizontal, bands of zoned composition carbonate. Figure 5. EDS spectra of two magnetites (bottom) embedded in carbonate matrix (top) in FIB sections. Carbonate contains detectable Mn while magnetite does not. Identical analysis time (1500 sec) and spot size were used. 